\title{
Effect of Environmental Stresses on Growth Pattern, Biofilm Formation and Biochemical Characteristics of Mycobacterium marinum CCUG20998
}

\author{
Mohammad Faezi Ghasemi, ${ }^{1,}$ Mina Nasiri Moslem,, and Mirsasan Mirpour ${ }^{1}$ \\ ${ }^{1}$ Faculty of Basic Sciences, Department of Microbiology, Lahijan Branch, Islamic Azad University, Lahijan, IR Iran \\ "Corresponding author: Mohammad Faezi Ghasemi, Faculty of Basic Sciences, Department of Microbiology, Lahijan Branch, Islamic Azad University, Lahijan, IR Iran. Tel: \\ +98-1342239042, Fax: +98-1342222605, E-mail: faezi_m@yahoo.com
}

Received 2015 June 04; Revised 2015 July 17; Accepted 2016 August 07.

\begin{abstract}
Background: Mycobacterium marinum is a ubiquitous, slow-growing nontuberclosis Mycobacterium (NTM), it can causes disseminated granulomatous infections in fish. Outbreaks in fisheries can be financially devastating and can also increase the chance of human exposure.

Objectives: The aim of this work was evaluating the effects of some environmental stresses on M. marinum CCUG 20998.

Methods: In this descriptive-analytic study M. marinum CCUG 20998 was subjected to different conditions of environmental stresses such as $\mathrm{pH}$, oxidative, osmotic pressure, and temperatures. The effects of stresses were studied on growth, biofilm formation, and cell division and biochemical characteristics of M. marinum CCUG 20998.The growth data were analyzed by measuring colony forming unit (CFU) using SPSS software version 19.

Results: The results showed that sodium chloride and hydrogen peroxide at $\% 10$ and $9600 \mathrm{ppm}$ concentrations inhibit. Marinum CCUG 20998 growths, respectively. Tolerance to $\mathrm{pH}=11$ and temperature at $82.5^{\circ} \mathrm{C}$ was detectable. Also, environmental stresses could affects on some biochemical characteristics of M. marinum CCUG 20998. Biofilm formation reduced upon using all stress conditions. Conclusions: Bacteria are able to adapt to dramatically different environments, In the case of mycobacteria, there is direct correlation between stress and pathogenicity. The results obtained from this study provided useful information on survival and tolerance of M. marinum CCUG 20998 to different environmental conditions. Survival under stress conditions might not reflect the in vivo situation where host factors also contribute to establishment of the organism during infection.
\end{abstract}

Keywords: Biofilm, Stresses, Biochemical, Characteristics, Mycobacterium marinum CCUG 20998

\section{Background}

The species in the genus Mycobacterium are acid fast and they inhabit in various environmental reservoirs such as, ground, tap water, soil, animals, and humans. They are grouped among the actinomycetes and include nonpathogens as well as highly successful pathogens e.g. Mycobacterium tuberculosis, Mycobacterium leprae and Mycobacterium ulcerans, the etiological agents of tuberculosis (TB; 10 million new cases per year develop active disease), leprosy and Buruli ulcer (the third most common mycobacterium infection), respectively [1-3].

Mycobacterium marinum is a close genetic relative of $M$. tuberculosis as they share more than $85 \%$ amino acid identity (a similarity of $99.3 \%$ in 16S rRNA sequence) and very closely related, both in its pathology and genetically, to $M$. ulcerans about more than share $99.6 \%$ amino acid identity. M. marinum is an attractive model system for identifica- tion, study of virulence factors, disease development, and drug resistance in $M$. tuberculosis. Moreover, M. marinum causes a systemic TB-like infection in ectodermic hosts e.g. fish and frogs. The infections caused by M. marinum in warm-blooded hosts, such as human, and poikilothermic animals, such as fishes and frogs, is indistinguishable with the infection caused by $M$.tuberculosis complex $[4,5]$. In comparison to M. tuberculosis, M. marinum grows with a relatively short doubling time and growth is limited at higher temperatures, making $M$. marinum easier to handle than e.g. M. tuberculosis and study in the laboratory [6].

Microbial population encounter different environmental stresses and usually adaptation to these stresses causes extended tolerance to multiple other lethal stressors. This phenomenon is defined as stress hardening, which refers to the increased resistance to lethal factors after adaptation. Like other bacteria, Mycobacterium spp. competes for nutrients, and they have developed sophis- 
ticated ways to adapt to different environmental stresses. Phosphorylation dependent signal systems and alternative $\sigma$-factors play essential roles as switches to alter gene expression patterns globally [7]. Although there are some progress in understanding the stress responses in $\mathrm{My}$ cobacterium spp. even at molecular level, but there are little information concerning the effects of environmental stresses on growth, cell division and biochemical characteristics of M. marinum.

\section{Objectives}

The aim of this study was evaluating the effects of some environmental stresses such as $\mathrm{pH}$, oxidative, temperatures and osmotic pressure on growth, biofilm formation, and biochemical characteristics of M. marinum CCUG 20998.

\section{Methods}

In this descriptive-analytic study $M$. marinum CCUG 20998 was subjected to different conditions of environmental stresses such as $\mathrm{pH}$, oxidative, osmotic pressure, and temperatures. The growth data were analyzed by measuring colony forming unit (CFU) using SPSS software version 19. All media and materials used in this study were obtained from (Merck Co. Darmstadt, Germany). Middlebrook 7H10 and 7H9 and Middlebrook OADC Enrichment were obtained from BD BBLTM Co. New Jersey USA.

\subsection{Bacterial Strain and Culture}

Mycobacterium marinum CCUG 20998 was obtained from culture collection, university of Goteborg, (CCUG, Goteborg, Sweden) used through this study. Lyophilized vial was cultured in Middlebrook 7H10 medium containing Middlebrook OADC enrichment. Incubation was performed at $30^{\circ} \mathrm{C}$ for 7 - 10 days until visible colony formation. For determining the mid-log phase, a single colony grown on Middlebrook 7H10 was inoculated in 7H9 Middlebrook medium. Optical density of growth was measured at $600 \mathrm{~nm}$ every 24 hours until the cells reach to mid-log phase. The cells at mid-log phase were harvested by centrifugation at 3,000 g at $4^{\circ} \mathrm{C}$ for 90 minutes. The harvested cells were washed three times in $5 \mathrm{~mL}$ of phosphate buffer $(\mathrm{PB})$ and resuspend in $10 \mathrm{~mL}$ of pre-chilled $\left(4^{\circ} \mathrm{C}\right) 0.1 \mathrm{M}$ phosphate buffer $(\mathrm{PB})$ and kept in refrigerator for evaluating the effects of different stress conditions.

\subsection{Condition Used for Different Stresses}

For evaluating different stresses, M. marinum CCUG 20998 cells in pre-chilled phosphate buffer was grown in 7H9 Middle brook broth medium to an optical density at $600 \mathrm{~nm}$ of $0.2-0.4$. For treating acidic or basic stresses, 50 micro liter of cell suspension inoculated in 7H9 Middlebrook medium having $\mathrm{pH}(3.0,5.0,7.0,9.0,1.0$ and 11. 0 ), $\mathrm{pH}$ values were adjusted using $0.5 \mathrm{M} \mathrm{HCl}$ and 1 $\mathrm{M} \mathrm{KOH}$, respectively. Incubation was continued for additional 24 hours before harvesting the cells. In order to assess osmotic pressure, M. marinum CCUG20998 cells in mid-log phase were transferred in to distilled water containing sodium chloride at concentrations of $0.0 \%, 2 \%, 4 \%$, $6 \%, 8 \%(\mathrm{w} / \mathrm{v})$ for two hours. For oxidative stress M. marinum CCUG20998 cells in mid-log phase were treated with $\mathrm{H}_{2} \mathrm{O}_{2}$ at concentrations of $600,1200,2400,4800$ and 9600 (ppm), for two hours. For heat stress the cultures were shifted to $32.5,42.5,52.5,62.5,72.5,82.5$ and $100^{\circ} \mathrm{C}$, respectively, and incubated for an additional 24 hours before harvesting, as described above (centrifugation was performed at $4^{\circ} \mathrm{C}$ ).The stress-free control was prepared by inoculating M. marinum CCUG 20998cells in pre-chilled phosphate buffer by transferring into pre-warmed 7H9 Middlebrook medium.

For evaluating the effect of each stresses on growth patterns, stressed cells were centrifuged at3000 rpm for 2 hours at $4^{\circ} \mathrm{C}$. Cell pellets were resuspended in 50 micro liter of $7 \mathrm{H} 9$ medium and inoculated in $100 \mathrm{~mL}$-Erlenmeyer containing $25 \mathrm{~mL}$ fresh 7H9 Middlebrook medium. Incubation was carried out for 120 hours.

The pattern of growth was measured (OD 600nm) with 24 hours intervals. Biochemical characteristics such as, Tween hydrolysis, nitrate reduction, aryl sulfatse, urease, telluride reduction, salt tolerance, semi quantitative catalase, niacin production, acid phosphatase, pyrazinamidase and other tests were evaluated and compared to nontreated cells.

\subsection{Estimation of Biofilm Formation by Stressed M. marinum CCUG 20998}

Biofilm formation was determined using Carter et al. method with some modifications $[8,9] .100 \mu$ Lof frozen stock culture was inoculated in $10 \mathrm{~mL}$ of fresh $7 \mathrm{H} 9$ medium with OADC and Tween 80 and incubated at $30^{\circ} \mathrm{C}$ with agitation for seven days. $200 \mu$ of the cell suspension were added to the wells of a 96- well flat bottom polystyrene micro titer plate in triplicate (MicroWell TM Plates Nunclon TM Nunc Nuncleon, Rosklide, Denmark), and incubated at $30^{\circ} \mathrm{C}$ without agitation in a sealed container with $20 \mathrm{~mL}$ sterile distilled water to prevent drying. Media without 
bacteria were used as negative controls on each plate. After incubation for two weeks, bacterial growth was determined by OD 600 measurement. The wells were washed once with $250 \mu \mathrm{L}$ tap water, and the remaining biofilm was stained using $250 \mu \mathrm{L} \mathrm{l}$ 1\% crystal violet, followed by 30 minutes incubation at room temperature. The wells were rinsed three or four times with tap water. The stained biofilm was resuspended in $250 \mu$ L ethanol: acetone 70:30. Finally, the amount of biofilm was measured at OD600. Results were presented as the medium value of triplicate, subtracting the medium value for the negative control.

\section{Results}

In this study exponential phase of Mycobacterium marinum CCUG 20998 cells were subjected to a variety stress conditions such as different sodium chloride, hydrogen peroxide concentrations, and different $\mathrm{pH}$ and temperature values. Figure 1 A shows growth pattern of M. marinum CCUG 20998 cells after treating with concentrations of 0.0 $\%, 2 \%, 4 \%, 6 \%, 8 \%(\mathrm{w} / \mathrm{v}$ ) sodium chloride in distilled water. The effect of different concentration of hydrogen peroxide, different temperatures, and $\mathrm{pH}$ on growth pattern of M. marinum CCUG 20998 is shown in Figure 1B, C and D, respectively. As shown in Figure $1 \mathrm{~A}$ sodium chloride at \%10 concentrations inhibited the growth of M. marinum CCUG 20998 cells. Hydrogen peroxide at 9600 ppm concentration and temperature at $62.5^{\circ} \mathrm{C}$ could inhibit M. marinum CCUG 20998 growth completely (Figure 1B and 1C), respectively. Maximum tolerance for $\mathrm{pH}$ was $\mathrm{pH}=11$ as shown in Figure 1D. The obtained results showed increased tolerance of M. marinum after treating to stress conditions in comparison to non-stressed cells. Table 1 shows the effects of different stress condition on biochemical characteristics of M. marinum CCUG 20998. As shown in the table the most effective stress conditions were $\mathrm{pH}=11$, sodium chloride $(\% 8 \mathrm{~W} / \mathrm{V}), \mathrm{H}_{2} \mathrm{O}_{2}$ at $4800 \mathrm{ppm}$ and temperature at $52.5^{\circ} \mathrm{C} . \mathrm{M}$. marinum CCUG 20998 growths was significant at $25-32^{\circ} \mathrm{C}$ temperature ranges. Pigmentation was lost at all stresses condition and cells showed sensitivity to isoniazid upon all stress conditions. Also heat stability, Tween hydrolysis, urea hydrolysis and acid phosphatase activity was negative in M. marinum CCUG 20998 cells treated with different stresses. Biofilm formation of M. marinum before and after stress conditions is shown in Figure 2. As shown there is good biofilm formation by M. marinum CCUG 20998 using $7 \mathrm{H} 9$ medium with Tween 80 at $32^{\circ} \mathrm{C}$. Biofilm formation by M. marinum CCUG 20998 reduced about $90 \%$ in conditions such as $\mathrm{pH}=11$ and hydrogen peroxide at 9600 ppm concentration.

\section{Discussion}

In this study, the effects of some environmental stresses on growth pattern, biofilm formation and biochemical characteristics by M. marinum CCUG20998 were investigated. Osmotic pressure, oxidative stress, different $\mathrm{pH}$ and temperatures were used. M. marinum CCUG 20998 can tolerate up to $8 \% \mathrm{~W} / \mathrm{V}$ sodium chloride concentration in $7 \mathrm{H} 9$ medium. Maximum tolerance up to $4800 \mathrm{ppm}$ $\mathrm{H}_{2} \mathrm{O}_{2}$ was detected in $M$. marinum CCUG 20998. Also, upon increasing oxidative and $\mathrm{pH}$ values more clumping formation detected.

Usually, for survival in the face of stress conditions bacteria may move by swimming using molecular motor and their flagella or may adapt to changes in their immediate vicinity by responding to the imposed stresses. This response is accomplished by changes in the patterns of gene expression for those genes whose products are required to combat the deleterious nature of the stress [10]. Osmolarity is an important criterion to distinguish between the external and internal associated environment around the bacteria [11]. Osmotic fluctuation alter torgur pressure, which can impair protein folding and metabolic activity [12]. Bacteria typically counteract such fluctuations through the compensatory accumulation or expulsion of compatible solutes that restore osmotic balance in the cells. In addition, pathogenic bacteria have virulenceassociated osmosensory mechanisms that are triggered at the transcriptional level $[13,14]$. It can be conclude that increasing sodium chloride concentration could decrease viability of cells and cell division patterns. Although the members in Mycobacterium genus are acid fast bacteria having a rigid complex cell wall, but it seems they cannot face to increasing osmotic pressure by synthesis of compatible solutes. Recently, Hatzios et al. 2013. reported that osmotic stress stimulates a signaling network in $M y$ cobacterium tuberculosis regulated by the eukaryotic-like receptor PknD (Ser /Thr protein kinase) [14]. Expression of the PknD substrate Rv0516c was highly induced by osmotic stress. Furthermore, Rv0516c disruption modified peptidoglycan thickness, enhanced antibiotic resistance, and activated genes in the regulon of the alternative $\sigma$ factor SigF (sigma factor F) .These findings identify an osmosensory pathway or chest rated by PknD, Rv0516c, and Sig F that enables adaptation to osmotic stress through cell wall remodeling and virulence factor production. The widespread occurrence of eukaryotic-like Ser/Thr protein kinases in bacteria, these proteins may play an important role in bacterial osmosensing.

$\mathrm{H}_{2} \mathrm{O}_{2}$ is a stable oxidant that reacts with most organic substrates. Many oxidizes in bacterial cells generate superoxide anion, which forms hydrogen peroxide by spon- 
A

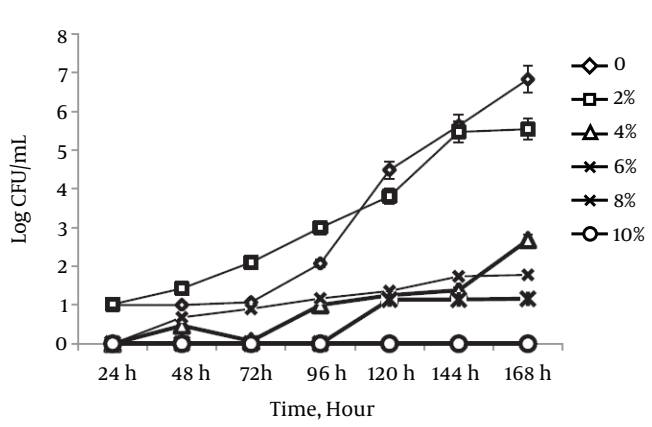

C

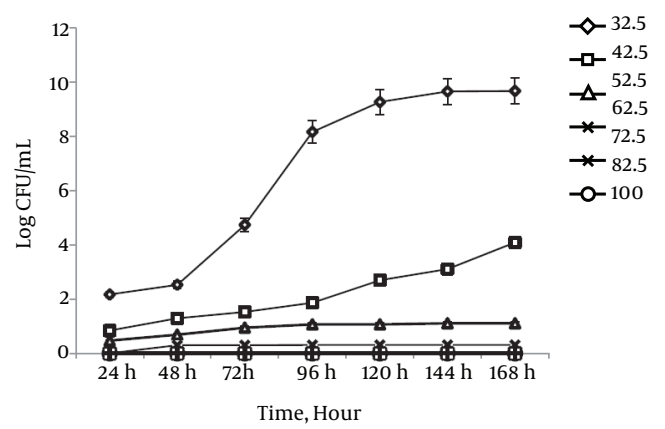

\section{B}

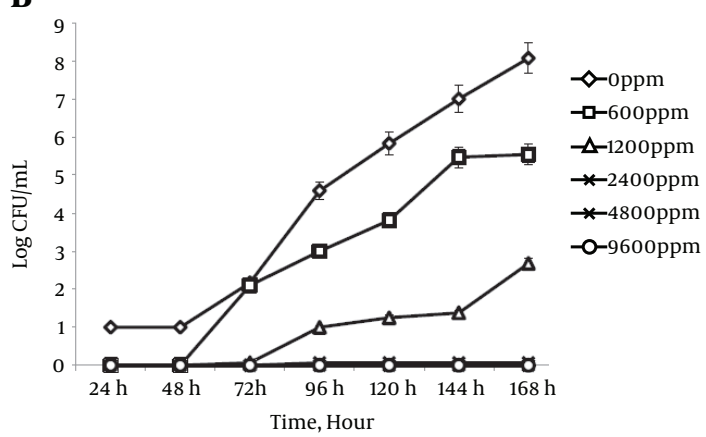

D

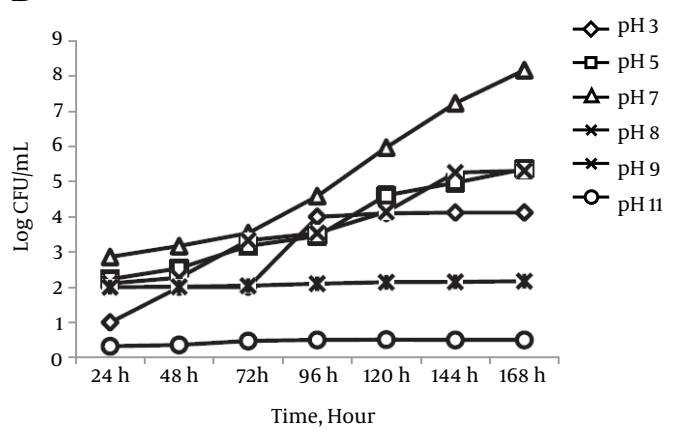

Figure 1. A-1D the Effects of Variety Stress Conditions Including Sodium Chloride, Hydrogen Peroxide, Different Temperatures and pH on Growth Pattern of M. marinum CCUG 2099

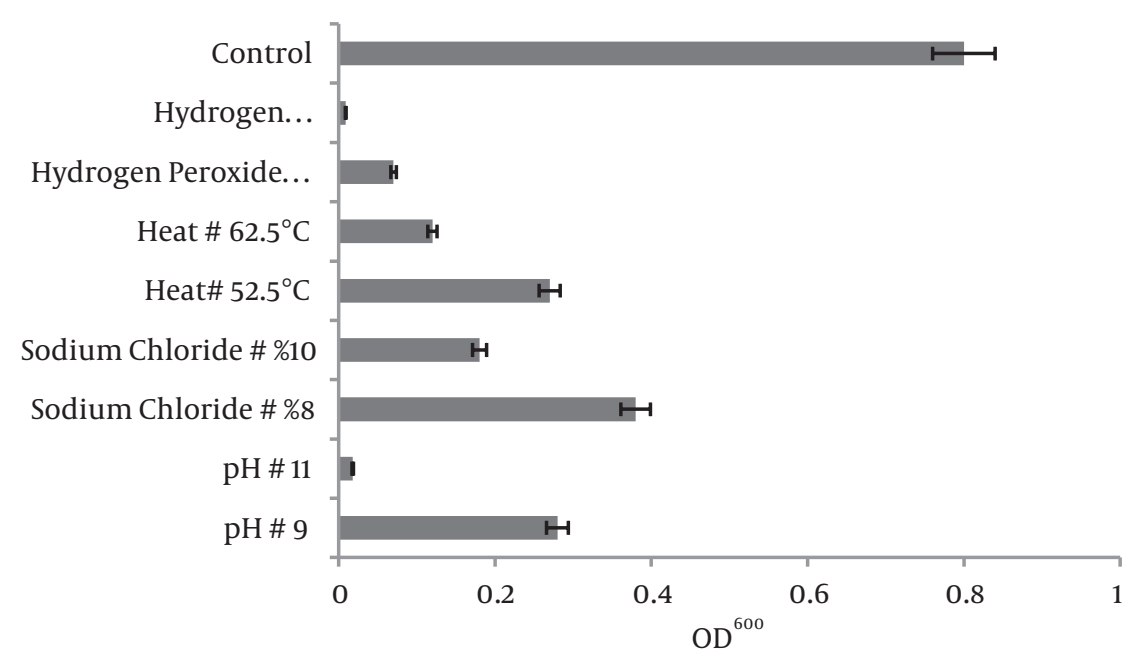

Figure 2. Comparison of Biofilm Formation by M. marinum CCUG 20998 Under Different Stress Conditions Including Sodium Chloride, Hydrogen Peroxide, Different Temperatures and $\mathrm{pH}$

taneous or catalyzed dismutation. It can cross cell membranes easily and the cytotoxicity is due to production of highly reactive hydroxyl radicals that are generated by Fenton's reaction. Because of the very short half-life of $\mathrm{OH}$ by product produced by $\mathrm{H}_{2} \mathrm{O}_{2}$ its reactions are diffusion limited, i.e., they take place practically at the site of generation. The cytotoxic nature of superoxide and peroxide, which are mild oxidizing agents, is due to their abil- 
Table 1. The Effects of Stress Conditions on Biochemical Characteristics of M. marinum CCUG $20998^{a}$

\begin{tabular}{|c|c|c|c|c|c|}
\hline \multirow[b]{2}{*}{ Characteristics } & \multicolumn{5}{|c|}{ Stress Condition } \\
\hline & $\mathbf{1}$ & 2 & 3 & 4 & 5 \\
\hline \multicolumn{6}{|l|}{ Growth at } \\
\hline $25-27^{\circ} \mathrm{C}$ & + & + & + & + & + \\
\hline $32^{\circ} \mathrm{C}$ & + & + & + & + & + \\
\hline $42.5^{\circ} \mathrm{C}$ & - & - & - & - & - \\
\hline $52.5^{\circ} \mathrm{C}$ & - & - & - & - & - \\
\hline Pigmentation & + & - & - & - & - \\
\hline \multicolumn{6}{|l|}{ Resistence to } \\
\hline Isoniazid, $1 \mu \mathrm{g} \mathrm{mL}^{-1}$ & M & $\neg-$ & - & - & - \\
\hline Isoniazid, $10 \mu \mathrm{g} \mathrm{mL}^{-1}$ & M & - & - & - & - \\
\hline $\mathrm{TCH}$ & + & - & - & - & - \\
\hline Hydroxyamine & + & + & + & + & + \\
\hline p-Nitrobenzoic acid & M & M & M & M & M \\
\hline Thiacetazone & $\mathrm{M}$ & M & M & M & M \\
\hline Oleate & $\mathrm{F}$ & $\mathrm{F}$ & - & $\mathrm{F}$ & $\mathrm{F}$ \\
\hline \multicolumn{6}{|l|}{ Catalase activity } \\
\hline$>45 \mathrm{~mm}$ & $\mathrm{~F}$ & - & - & - & - \\
\hline Heat stable & + & - & - & - & - \\
\hline Tween 80 hydrolysis & + & - & - & - & - \\
\hline Urease & + & - & - & - & - \\
\hline Niacin production & - & - & - & - & - \\
\hline Nitrate reduction & - & - & - & - & - \\
\hline Acid phosphatase & + & - & - & - & - \\
\hline Arylsulphatase, 3 day & $\mathrm{F}$ & - & - & - & - \\
\hline Pyrazinamidase, 7 days & M & - & - & - & - \\
\hline
\end{tabular}

ity to generate intracellular OH. DNA which is damaged by such free radicals contains a multiplicity of base and sugar derivatizations and most of these are mutagenic. Attack at a sugar leads to sugar fragmentation as well as loss of base resulting in strand break. Radicals can also damage the bases of the DNA such as ring-saturated thymines, hydroxyl methyl uracil, thymine fragments and adenine ring opened products $[15,16]$. The results in this study showed that upon increasing oxidative and $\mathrm{pH}$ values more clump formation was detected. So, it can be concluded that clump formation is one of the ways that the species in the genus Mycobacterium could response in harsh environments such as exposing to different stress isolated M. marinum from biofilm in water distribution systems, also quantified the biofilm formation by M. marinum and the effect of antimicrobial agents were evaluated on biofilm formation by this organism $[17,18]$.

Bacterial responses to different stresses conditions have been studied mostly under in vitro conditions. Although the results obtained from these studies have provided useful information on survival under stress condi- tions and expression of different genes, they might not truly reflect the in vivo situation where host factors also contribute to establishment of the organism during infection. For a better understanding of interaction between host and parasite it is desirable to delineate the bacterial functions that are specifically expressed under in vivo conditions and to assign their role in pathogenesis. Several approaches are now being adopted to identify in vivo expressed genes and examine their functions. The results from such analysis will furnish a clear picture of how animal or human pathogens adapt themselves to in vivo stress situations and provide a better insight into the molecular basis of pathogenicity.

\section{Acknowledgments}

We would like to thanks vice-presidency of research affairs of Islamic Azad University, Lahijan branch for providing facilities during this project. This research was performed based on a master of science (MSc) thesis No. 20230507922028 addressed to Mina Nasiri Moslem under 
supervision of Dr. Mohammad Faezi Ghasemi.

\section{Footnotes}

Authors' Contribution: All authors had equal role in design, work, and performing the lab works.

Funding/Support: This study was supported by Islamic Azad University, Lahijan branch.

\section{References}

1. Demangel C, Stinear TP, Cole ST. Buruli ulcer: reductive evolution enhances pathogenicity of Mycobacterium ulcerans. Nat Rev Microbiol. 2009;7(1):50-60. doi:10.1038/nrmicro2077. [PubMed: 19079352].

2. Russell DG. Who puts the tubercle in tuberculosis?. Nat Rev Microbiol. 2007;5(1):39-47. doi: 10.1038/nrmicro1538. [PubMed: 17160001].

3. Sasaki S, Takeshita F, Okuda K, Ishii N. Mycobacterium leprae and leprosy: a compendium. Microbiol Immunol. 2001;45(11):729-36. [PubMed: 11791665].

4. Wayne LG, Kubica GP. The mycobacteria. In: Sneath PH, Mair NS, Sharpe ME, Holt JG, editors. Bergys manual of systematic bacteriology. United States: Williams and Wilkins Baltimore; 1986. pp.1436-57.

5. Gey van Pittius NC, Sampson SL, Lee H, Kim Y, van Helden PD, Warren RM. Evolution and expansion of the Mycobacterium tuberculosis PE and PPE multigene families and their association with the duplication of the ESAT-6 (esx) gene cluster regions. BMC Evol Biol. 2006;6:95. doi: 10.1186/1471-2148-6-95. [PubMed: 17105670].

6. Ramakrishnan L. Using mycobacterium marinum and its hosts to study tuberculosis. Curr Sci. 2004;86(1):82-92.

7. Storz G, Hengge R. Bacterial stress responses American Society for Microbiology Press; 2010. Available from: https://books.google. com/books?hl=en\&lr=\&id=VwOsBAAAQBAJ\&oi=fnd\&pg= PT10\&dq $=$ Storz $+\mathrm{G},+$ Hengge $+\mathrm{R} .+$ Bacterial+stress + responses: + American+Society+for+Microbiology+Press $\% 3 B+2010 \&$ ots $=$ QX50q5Coh6\&sig=3Uo_0iPYlxAQnuYeOactBe0fe9s\#v= onepage $\& \mathrm{q} \& \mathrm{f}=$ false.
8. Carter G, Wu M, Drummond DC, Bermudez LE. Characterization of biofilm formation by clinical isolates of Mycobacterium avium. Med Microbiol. 2003;52(Pt 9):747-52. doi: 10.1099/jmm.0.05224-0. [PubMed: 12909649].

9. Johansen TB, Agdestein A, Olsen I, Nilsen SF, Holstad G, Djonne B. Biofilm formation by Mycobacterium avium isolates originating from humans, swine and birds. BMC Microbiol. 2009;9:159. doi: 10.1186/1471-2180-9-159. [PubMed: 19660141].

10. Mitchell JG, Kogure K. Bacterial motility: links to the environment and a driving force for microbial physics. FEMS Microbio Ecol. 2006;55(1):3-16. doi: 10.1111/j.1574-6941.2005.00003.x. [PubMed: 16420610].

11. Chowdhury R, Sahu GK, Das J. Stress response in pathogenic bacteria. Jbiosci. 1996;21(2):149-60.

12. Sleator RD, Hill C. Bacterial osmoadaptation: the role of osmolytes in bacterial stress and virulence. FEMS Microbiol Rev. 2002;26(1):49-71. [PubMed: 12007642].

13. Gao H, Zhang Y, Han Y, Yang L, Liu X, Guo Z, et al. Phenotypic and transcriptional analysis of the osmotic regulator OmpR in Yersinia pestis. BMC Microbiol. 2011;11:39. doi: 10.1186/1471-2180-11-39. [PubMed: 21345178].

14. Hatzios SK, Baer CE, Rustad TR, Siegrist MS, Pang JM, Ortega C, et al. Osmosensory signaling in Mycobacterium tuberculosis mediated by a eukaryotic-like Ser/Thr protein kinase. Proc Natl Acad Sci U S A. 2013;110(52):E5069-77. doi: 10.1073/pnas.1321205110. [PubMed: 24309377].

15. Gupta S, Chatterji D. Stress responses in mycobacteria. IUBMB Life. 2005;57(3):149-59. doi: 10.1080/15216540500090611. [PubMed: 16036577 .

16. Imlay JA. Pathways of oxidative damage. Annu Rev Microbiol. 2003:57:395-418. doi: 10.1146/annurev.micro.57.030502.090938. [PubMed: 14527285].

17. Bardouniotis E, Ceri H, Olson ME. Biofilm formation and biocide susceptibility testing of Mycobacterium fortuitum and Mycobacterium marinum. Curr Microbiol. 2003;46(1):28-32. doi: 10.1007/s00284-0023796-4. [PubMed: 12432460].

18. Falkinham JO, Norton CD, LeChevallier MW. Factors influencing numbers of Mycobacterium avium, Mycobacterium intracellulare, and other Mycobacteria in drinking water distribution systems. Appl Environ Microbiol. 2001;67(3):1225-31. doi: 10.1128/AEM.67.3.1225-1231.2001. [PubMed: 11229914]. 